\title{
Erster Fachkongress der Gesellschaft für Hygiene, Umweltmedizin und Präventivmedizin (GHUP) gemeinsam mit der 10. Jahrestagung des Landesinstituts für den Öffentlichen Gesundheitsdienst (lögd) NRW
}

\author{
First Congress of the Society of "Hygiene, Umweltmedizin und Präventivmedizin (GHUP)" together with \\ the $10^{\text {th }}$ Annual Meeting of the "Landesinstitut für den Öffentlichen Gesundheitsdienst (lögd) NRW"
}

Autoren

Institute

\section{Hornberg', R. Fehr ${ }^{2}$}

Arbeitsgruppe 7 „Umwelt und Gesundheit“, Fakultät für Gesundheitswissenschaften, Universität Bielefeld

Landesinstitut für Gesundheit und Arbeit, Bielefeld
Bibliografie

DOI $10.1055 / \mathrm{s}-2007-995617$

Akt Dermatol 2008; 34:

98-100 @ Georg Thieme

Verlag KG Stuttgart · New York ISSN 0340-2541

Korrespondenzadresse

Prof. Dr. Claudia Hornberg

Arbeitsgruppe 7 „Umwelt

und Gesundheit“", Fakultät für

Gesundheitswissenschaften

Universität Bielefeld

Postfach 100131

33501 Bielefeld

claudia.hornberg@

uni-bielefeld.de
Global wachsende umweltbedingte Krankheitslasten und umweltassoziierte Gesundheitsstörungen zählen zu den größten Herausforderungen der kommenden Jahre. Sie berühren im Hinblick auf ihre Komplexität das gesamte Tätigkeitsspektrum von Hygiene, Umwelt- und Präventivmedizin und erfordern darüber hinaus den Brückenschlag zu weiteren Fachgebieten wie der Arbeitsmedizin, der Dermatologie/Allergologie und der Psychosomatik sowie die Kooperation mit den außerhalb der Medizin verorteten natur- und sozialwissenschaftlichen Disziplinen. Um die gegenwärtigen und anstehenden Aufgaben auf nationaler und internationaler Ebene bewältigen zu können, wird es künftig mehr denn je darauf ankommen, fachliche Kompetenzen interdisziplinär zu bündeln und im Sinne von Environmental Health und Public Health zu nutzen bzw. weiterzuentwickeln.

Ein erster bedeutsamer Schritt in der Umsetzung dieses Erfordernisses wurde im Jahr 2005 mit dem Zusammenschluss der beiden wissenschaftlichen Fachgesellschaften im Bereich der Umweltmedizin ISEM (International Society of Environmental Medicine) und GHU (Gesellschaft für Hygiene und Umweltmedizin) eingeleitet. Ein wichtiges Anliegen der neu etablierten Gesellschaft für Hygiene, Umweltmedizin und Präventivmedizin (GHUP) ist es, die Environmental Health und Public Health Sciences als ein interdisziplinäres, international anschlussfähiges Fachgebiet in Deutschland weiterzuentwickeln und zu positionieren. Daneben verfolgt die GHUP eine Stärkung der Facharztausbildung für Hygiene und Umweltmedizin sowie insgesamt die Förderung umweltmedizinischer Inhalte in Aus-, Fort- und Weiterbildung.

Nach fast zwei Jahren organisatorischer und personeller Aufbauarbeit hatte die GHUP im Jahr 2007 eine Premiere. In Kooperation von GHUP, dem Landesinstitut für den Öffentlichen Gesundheitsdienst (lögd) Nordrhein-Westfalen sowie der Fakultät für Gesundheitswissenschaften der Universität Bielefeld fand vom 22. bis 24. November 2007 in Bielefeld der erste Fachkongress der GHUP gemeinsam mit der 10. Jahrestagung des Landesinstituts für den Öffentlichen Gesundheitsdienst (lögd) NRW statt. Rund 350 Expertinnen und Experten aus unterschiedlichen umwelt- und gesundheitsbezogenen Wissenschaftsbereichen und Praxisfeldern aus Deutschland, Österreich und der Schweiz reisten an, um sich an drei Tagen in einem fachübergreifenden Wissens-, Erfahrungs- und Informationsaustausch dem Thema „Problemlösungs- und Präventionsstrategien für den umweltbezogenen Gesundheitsschutz" zu widmen.

Mit der Wahl des Tagungsthemas wurde bewusst Wert darauf gelegt, die klassische Pathogeneseund Risikoperspektive zu erweitern und den gesundheitswissenschaftlichen und humanökologischen Ressourcenansätzen angemessenen Raum zu geben. Plenarveranstaltungen, Vorträge und Round Table-Diskussionen boten hierzu ebenso Gelegenheit wie eine begleitende Industrieausstellung. Forschungsgruppen sowie der wissenschaftliche Nachwuchs nutzte darüber hinaus intensiv die Möglichkeit, die eigene Arbeit mit einem Poster zu präsentieren. Höhepunkt am Abend des zweiten Kongresstages war demzufolge die zentrale Poster-Session, die angesichts der hervorragenden Qualität und der Vielzahl der präsentierten Poster Fortbildungscharakter hatte. Die nachfolgende Prämierung der drei besten Beiträge, für die großzügige Geldpreise ausgesetzt waren, stellte die Jury vor eine schwierige Aufgabe.

Mit einer vergleichbaren Herausforderung sah sich bereits einige Monate zuvor das Wissenschaftliche Komitee im Rahmen der Programmgestaltung und der Auswahl der Beiträge konfrontiert. Das Resultat der Bemühungen spiegelte sich in mehr als 170 Beiträgen in 22 Parallelveranstaltungen mit einem ausgesprochen breiten 
Themenspektrum wider, das in seiner inhaltlichen Ausrichtung unterschiedlichen Dimensionen einer umweltbezogenen Perspektive auf Gesundheit und Krankheit zuzuordnen war. Die Exposition gegenüber Umweltnoxen wie chemischen Schadstoffen, physikalischen und mikrobiologischen Faktoren, aber auch sozialen Faktoren wurde mit Blick auf zentrale Lebensbereiche wie Wohnung, Arbeitsplatz, Schule und Einrichtungen der Gesundheitsversorgung etc. thematisiert. Daneben standen aktuelle Fakten, Analyse- und Bewertungsszenarien sowie Handlungsperspektiven in Bezug auf ausgewählte Umweltmedien wie Trinkwasser, Außen- und Innenraumluft als Noxenträger im Fokus der Tagungsthemen. Auf große Resonanz trafen die diversen Beiträge zu innovativen und weiterentwickelten umweltepidemiologischen und umweltmedizinischen Methoden und Instrumenten, z.B. zur quantitativen Abschätzung umweltbedingter Risiken für die Gesundheit der Bevölkerung („environmental burden of disease“). Die Dringlichkeit weitergehender, spezifizierter Analysen zur Aufklärung der Zusammenhänge zwischen Umwelt und Gesundheit (z.B. Identifizierung bevölkerungsspezifischer Expositionsunterschiede) wurde am Beispiel des Klimawandels mit seinen potenziellen Folgen für die Humangesundheit illustriert. Angesichts der Globalisierung umweltbezogener Gesundheitsbelastungen zeigte sich darüber hinaus die wachsende Relevanz von Environmental Health und Public Health als „Wegweiser“ für die Orientierung am vorsorgenden umweltbezogenen Gesundheitsschutz im Rahmen politischer Prioritätensetzung und forschungsstrategischer Ausrichtung. Anzustreben und einzufordern ist demnach eine stärkere Beteiligung umweltmedizinischer Expertise, z. B. des öffentlichen Gesundheitsdienstes, in der Bewertung gesundheitlicher Chancen und Risiken anstehender politischer und planerischer Entscheidungen, um ad hoc-Maßnahmen der Gefahrenabwehr oder symptomorientierte end-of-pipe-Strategien möglichst zu vermeiden.

Gefahrenabwehr und Symptomorientierung erweisen sich speziell mit Blick auf besonders vulnerable Bevölkerungsgruppen wie Schwangere, alte Menschen, sozial Benachteiligte sowie Kinder und Jugendliche als unzureichend. Dies legten unter anderem Präsentationen zu aktuellen Ergebnissen epidemiologischer Untersuchungen wie Kinder-Umwelt-Survey (KUS) nahe sowie Beiträge, die auf Umweltexpositionen bei Kindern und Jugendlichen als besondere Zielgruppe für umweltbezogene Prävention und Gesundheitsförderung fokussierten. Das daraus abzuleitende Erfordernis einer Zielgruppenorientierung zog sich implizit durch das gesamte Spektrum der Tagungsbeiträge und umfasste auch die Thematisierung der vielfach noch unzureichenden Integration der Gender-Dimension und eines geschlechterdifferenzierenden bzw. geschlechtersensiblen Vorgehens in Hygiene, Umwelt- und Präventivmedizin.

Dass Zielgruppenorientierung in der umweltbezogenen Prävention weitaus mehr beinhaltet als die Berücksichtigung von Alter und Geschlecht, demonstrierten die Themenbeiträge in der Session „Umweltgerechtigkeit“, einem Forschungs- und Handlungsfeld an der Schnittstelle von Environmental Health und Public Health Sciences. Die Verbindung von Fragen sozialer Gerechtigkeit mit der Verteilung gesundheitsschädlicher Umweltbelastungen und gesundheitsfördernder Umweltressourcen findet zunehmend auch in den deutschen Wissenschaftskontext Eingang. Anknüpfend an den US-amerikanischen „Environmental Justice“-Diskurs erweitert die Umwelt(un)gerechtigkeitshypothese die naturwissenschaftlich geprägte Umweltdefinition um die Relevanz der Lebensumwelt mit ihren sozialen Realitäten für „environmental health“. Angesichts des sich vergrößern- den sozialen Gefälles in den wohlhabenden Industrienationen und der gleichzeitigen Zunahme von Erkrankungen, für die auch Umwelteinflüsse (z.B. Lärm, Feinstaub) als relevant anzunehmen sind, ist Umwelt(un)gerechtigkeit eine zentrale gesamtgesellschaftliche Herausforderung, der sich auch Environmental Health und Public Health stellen müssen.

Des Weiteren zeichnete sich in zahlreichen Beiträgen der Bedarf nach erweiterten und verbesserten Dialogstrukturen in der Gesundheitsvorsorge, im umweltbezogenen Gesundheitsschutz sowie in der Qualitätssicherung ab. Dass diese beispielsweise durch die Förderung kommunaler Zusammenarbeitsstrukturen sowie durch eine grenzüberschreitende Zusammenarbeit zu realisieren sind, davon konnten sich die Teilnehmerinnen und Teilnehmer in einem Workshop zum EUREGIO MRSA-net-Projekt, einem grenzübergreifenden deutsch-niederländischen Qualitätsverbund, überzeugen. Die Senkung der MRSA (Methicillin-resistenter Staphylococcus aureus)-Rate auf niederländisches Niveau sowie die Kontrolle der von Deutschland ausgehenden MRSA-Ausbreitung in die niederländische Grenzregion soll durch Kooperation und Informationsaustausch innerhalb der EUREGIO dazu beitragen, die bestehenden Hindernisse im grenzüberschreitenden Verkehr von Patienten und Personal im Gesundheitswesen zu senken.

Dieser innovative Ansatz war nur einer von vielen vorbildlichen Beispielen aus Forschung und Praxis, die während des dreitägigen Fachkongresses präsentiert wurden. In der Gesamtschau zeichnete sich deutlich ab, dass eine inter- und transdisziplinäre Betrachtung aus den Einzeldisziplinen eine zentrale Voraussetzung darstellt, um der Komplexität umweltbezogener Gesundheit zu entsprechen. In diesem Sinne hatte auch der Entschluss, die 1. Jahrestagung der GHUP mit der 10. Jahrestagung des LÖGD gemeinsam zu veranstalten, einen symbolischen Charakter. Er reflektiert einerseits das Bestreben von Hygiene, Umwelt- und Präventivmedizin, den Dialog mit Nachbardisziplinen zu suchen und gemeinsam neue theoretische und praxisorientierte Perspektiven zu entwickeln. Auf der anderen Seite verweist er auf die zahlreichen Schnittstellen, die zwischen der Arbeit des ÖGD, etwa in den Bereichen Gesundheitsberichterstattung, Umsetzung von Gesundheitszielen, Infektionsschutz, Mitwirkung an räumlicher Planung im Rahmen von Health Impact Assessment (HIA) etc. und den Environmental Health und Public Health Sciences bestehen. Dass sich die Anstrengungen nicht darauf beschränken dürfen, Umweltbelastungen zu reduzieren und Gefahren zu mindern, sondern dass mit gleichen Bemühungen sowohl verhaltens- als auch lebensumweltbezogene Gesundheitsressourcen zu stärken und $\mathrm{zu}$ fördern sind, war ein zentrales Anliegen dieses Fachkongresses, das die weitere Arbeit der GHUP begleiten wird.

Im Sinne eines Brückenschlags zwischen den unterschiedlichen Fachrichtungen wäre es wünschenswert, für Hygiene, Umweltund Präventivmedizin sowie für Dermatologie/Allergologie gleichermaßen relevante und aktuelle Themen z. B. im Rahmen von gemeinsamen Fachtagungen, Arbeitsgruppen, Weiterbildungen, Kompetenznetzwerken etc., zu erörtern und fachliche Berührungspunkte, Überschneidungen sowie auch Unterschiede in Forschung, Lehre und Praxis zu nutzen. Hier bieten sich vor allem Krankheitsbilder an, die durch eine Überlagerung (umwelt-)dermatologischer und umweltmedizinischer/-hygienischer Fragestellungen charakterisiert sind, so z. B. atopische Erkrankungen wie Asthma, Heuschnupfen, Hautveränderungen durch chemische, thermische, mechanische oder Strahleneinwirkung, atopische Dermatiden, Prävention und Therapie von 
Methicillin-resistentem Staphylococcus aureus (MRSA) etc. Hinzu kommen neue Fragestellungen wie die zu erwartenden Effekte des Klimawandels auf die Morbidität der Bevölkerung, die in weiten Teilen auch dermatologische/allergologische Arbeitsfelder berühren (z.B. der potenzielle Einfluss von Klimaveränderungen auf die Prävalenz atopischer Erkrankungen bei Kindern und Erwachsenen).

In all diesen Themenfeldern lassen sich zahlreiche Anknüpfungspunkte zwischen den in der GHUP vertretenen Fachdisziplinen und der Dermatologie/Allergologie finden, sodass der Ausbau eines fachübergreifenden Austauschs sowie eine Stärkung des Informationsflusses zwischen den verschiedenen Akteuren in Forschung und Praxis auf einer gemeinsamen inter- und transdisziplinären Plattform ein wünschenswertes und anzustrebendes Ziel wäre. In diesem Sinne würden wir uns im Namen der GHUP freuen, während der 2. Jahrestagung der GHUP, die vom 1.-4. Oktober 2008 unter der Leitung von Prof. Dr. Dr. Egon Marth in der Stadt Graz (Österreich) stattfinden wird, wieder möglichst viele Fachkolleginnen und Fachkollegen aus der Dermatologie/Allergologie begrüßen zu dürfen.

\section{Buchbesprechung}

Vakuumtherapien akuter und chronischer Wunden praktische Durchführung und wissenschaftliche Hintergründe

\section{Dissemond J, Horch RE}

Bremen: UNI-MED, 2007. 109 S., 153 überwiegend farbige

Abb., 13 Tab. Geb. 39,80€

ISBN 978-3-8374-1002-0

Die Herausgeber Herr Priv.-Doz. Dr. med. Joachim Dissemond von der Klinik für Dermatologie, Venerologie und Allergologie des Universitätsklinikums Essen und Herr Univ.-Prof. Dr. med. Raymund E. Horch von der Klinik für Plastische und Handchirurgie des Universitätsklinikums Erlangen, und ihre acht Co-Autoren stellen im vorliegenden Buch die praktische Durchführung sowie die wissenschaftlichen Hintergründe der Vakuumtherapie von akuten und chronischen Wunden dar. Das Buch soll nach ihren Vorstellungen ein „kurz gefasster Leitfaden für die tägliche Praxis“ sein und die interdisziplinäre Zusammenarbeit herausstellen.

Das Werk umfasst 11 Abschnitte untergliedert in insgesamt 43 Kapitel. Es beginnt mit einer Einführung in die Thematik. Nach einem kurzen historischen Diskurs werden die praktische Durchführung, Vor- und Nachteile der Vakuumtherapie sowie Indikationen und Kontraindikationen dargelegt. In den folgenden Abschnitten wird die Anwendung der Vakuumtherapie in den verschiedenen Fachdisziplinen wie Chirurgie, Orthopädie und Unfallchirurgie, Thoraxchirurgie und in der Dermatologie erläutert. Es folgt die Darstellung der Vakuumtherapie im Rahmen des plastisch-chirurgischen Versorgungskonzepts bei Diabetischem Fußsyndrom sowie in der Gefäßchirurgie zur Ergänzung der peripheren Revaskularisation bei Diabetikern. Hieran schließt sich der Abschnitt zum Thema Bakterien in chronischen Wunden unter Vakkumtherapie mit einer Darstellung der klinischen Studien und einer Diskussion der Studienergebnisse an. Es folgt der Abschnitt zur Evidenzlage der Vakuumtherapie, wobei die Herausgeber bereits im Vorwort darauf hinweisen, dass die auf empirischer Grundlage entstandenen klinischen Erfolge noch im Sinne einer evidenzbasierten Medizin bewiesen werden müssen. Das Buch endet mit dem Abschnitt über Praktische Tipps und Tricks bei Anwendung der Vakuumtherapie.

Mit diesem Buch liegt ein kurz gefasstes Werk zur Vakuumtherapie vor, welches doch alle für die tägliche Praxis relevanten Details und theoretischen Grundlagen liefert. Für den Dermatologen besonders interessant natürlich die Kapitel zur Vakuumtherapie bei Ulcus cruris, zur Einheilung von Spalthauttransplantaten nach Fixierung mit Vakuumtherapie und zum Wundrandschutz unter Vakuumtherapie. Ergänzt durch die Kapitel zum Umgang mit irritierter Wundumgebung, zu Schmerzen unter der Therapie und zur Wundinfektion gibt das Werk eine praktische Hilfestellung für die stationäre Handhabung der Vakuumtherapie und ist daher sehr empfehlenswert für den klinischen Alltag.

Carolin Imhoff, Wiesbaden 